\title{
Tricuspid valve surgery: The past 10 years from the Nationwide Inpatient Sample (NIS) database
}

\author{
Christina M. Vassileva, MD, John Shabosky, BA, Theresa Boley, MSN, Stephen Markwell, MA, and \\ Stephen Hazelrigg, MD
}

Objectives: The purpose of this study was to examine the trends in tricuspid valve surgery over time.

Methods: We used 10 years (1999-2008) of NIS data to examine the population of patients undergoing tricuspid valve repair or replacement (ICD-9-CM codes 35.14, 35.27, and 35.28).

\begin{abstract}
Results: We identified 28,726 admissions for tricuspid valve surgery. The total number of tricuspid procedures more than doubled over the 10- year period (1712 cases in 1999 vs 4072 cases in 2008). Although the absolute number of repairs and replacements increased over time, the tricuspid repair rate increased whereas there was a corresponding decrease in tricuspid replacement rate. Isolated tricuspid valve surgery accounted for $20 \%$ of the total tricuspid cases, whereas tricuspid surgery as a concomitant procedure to other cardiac operations accounted for the remaining $80 \%$. There was a trend toward increased use of tissue over mechanical valves for tricuspid replacement. Overall hospital mortality was $10.6 \%$. Over time, mortality decreased significantly for both repair and replacement. Concomitant tricuspid replacement was associated with significantly higher hospital mortality than was isolated tricuspid replacement $(16.1 \%$ vs $10.1 \% ; P=.0001)$.
\end{abstract}

Conclusions: There has been a dramatic increase in tricuspid interventions over time. This has been associated with an increase in tricuspid repair rates as well as use of bioprostheses for tricuspid replacement. The majority of tricuspid operations are performed concomitantly to other cardiac procedures. Mortality for tricuspid valve surgery remains considerable and significantly higher for replacement than for repair. (J Thorac Cardiovasc Surg 2012;143:1043-9)

Significant advances in surgical care for patients with valvular heart disease have taken place in the United States over the recent past. Within the area of mitral valve surgery, there has been an increased use of repair as the preferred surgical approach for the correction of mitral valve disease. ${ }^{1}$ In the area of aortic valve disease, outcomes have shown considerable improvement with decreased mortality and major perioperative morbidity. ${ }^{2}$ Tricuspid valve (TV) surgery is performed much less often than surgery on the mitral or aortic valves. Consequently, published reports have been limited by small numbers, typically from single institutions. In addition, there has been ongoing debate regarding the optimal surgical approach for TV disease (repair vs replacement) $)^{3,4}$ and type of prosthesis used (mechanical vs bioprosthesis). ${ }^{5}$ The purpose of our investigation was to review the trends in demographics, case mix, procedure selection, and hospital outcomes of patients

\footnotetext{
From the Division of Cardiothoracic Surgery, Southern Illinois University School of Medicine, Springfield, Ill.

Disclosures: Authors have nothing to disclose with regard to commercial support. Received for publication April 15, 2011; revisions received June 9, 2011; accepted for publication July 11, 2011; available ahead of print Aug 29, 2011.

Address for reprints: Christina M. Vassileva, MD, Division of Cardiothoracic

Surgery, Southern Illinois University School of Medicine, 701 N First St-Room

D318, Springfield, IL 62794-9638 (E-mail: cvassileva@ siumed.edu).

$0022-5223 / \$ 36.00$

Copyright (c) 2012 by The American Association for Thoracic Surgery

doi:10.1016/j.jtcvs.2011.07.004
}

undergoing TV surgery over the period from 1999 to 2008 using data from the largest all-payer database in the United States, the Nationwide Inpatient Sample (NIS), Healthcare Cost and Utilization Project (HCUP), Agency for Healthcare Research and Quality.

\section{METHODS \\ Database}

The NIS is a stratified probability sample developed as part of the HCUP funded by the Agency for Healthcare Research and Quality. It includes data from roughly $20 \%$ of hospital admissions in the United States. So that sampling bias can be minimized, the NIS is stratified by geographic region, urban versus rural location, teaching status, and hospital bed size. In other words, hospitals are divided into homogeneous subgroups with respect to these characteristics. For example, if a random $20 \%$ sample of hospital admissions is obtained, there may be an overrepresentation of urban hospitals or hospitals from certain geographic regions with a corresponding underrepresentation of rural hospitals or hospitals from other geographic regions. With a stratified probability sample, hospitals within each of the different strata in the overall sample have an equal probability representation in the selected $20 \%$ sample. The data are weighted to represent the entire inpatient population. The database contains deidentified patient data including up to 15 procedure and 15 diagnostic codes according to the International Classification of Diseases, Ninth Edition, Clinical Modification (ICD-9 $\mathrm{CM})$. The NIS is the largest all-payer database and is used for analysis of trends in health care utilization, access, charges, quality, and outcomes for both research and policy-making purposes (HCUP databases). The NIS shares many similarities with the Medicare database, including the same ICD-9-CM coding system for procedures and diagnoses. Unlike the Medicare database, however, which includes data on a single payer and as a result tends to include patients 65 years or older, the NIS includes all payers and 


\section{Abbreviations and Acronyms \\ $\mathrm{CABG}=$ coronary artery bypass grafting \\ HCUP $=$ Healthcare Cost and Utilization Project \\ ICD-9- = International Classification of Diseases, \\ CM Ninth Edition, Clinical Modification \\ NIS = Nationwide Inpatient Sample \\ $\mathrm{TV}=$ tricuspid valve}

The majority of the TV operations $(98.5 \%)$ were performed in urban hospitals, and $72.5 \%$ were performed in teaching hospitals. A greater proportion of replacements $(77.4 \%)$ were performed in teaching hospitals than repairs (70.7\%; $P=.0023)$. When broken down by region, $22.5 \%$ of the total procedures were performed in the Northeast, $25.4 \%$ in the South, $32.4 \%$ in the Midwest, and $19.7 \%$ in the West. A higher proportion of patients from the South underwent repair $(P=.0137)$ and a higher proportion of patients from the West underwent replacement $(P=.0023)$. The number of TV operations increased with increasing hospital bed size. For example, $79.0 \%$ of operations were performed in large hospitals whereas only $3.9 \%$ were done in small hospitals. However, there was no significant difference between the repair and replacement groups with respect to hospital bed size.

\section{Sample Selection}

The NIS database was searched from 1999 to 2008 to identify patients with TV repair (ICD-9-CM code 35.14) or replacement (ICD-9-CM codes 35.27 and 35.28). Patients less than 30 years of age and those with congenital heart disease were excluded. The final patient population consisted of 5808 observations representing 28,726 admissions for TV surgery (after appropriate weighting). Data are reported as number of admissions.

\section{Statistical Analysis \\ Descriptive statistics including frequencies, percents, means, medians, and interquartile ranges are presented. Group comparisons were carried out using $\chi^{2}$ tests of independence, independent groups $t$ tests, and median tests. Cochran-Armitage tests were used to test for trends across the years. All analyses were performed using SAS version 9.2 which allows for weighting to reflect the stratified sampling scheme used in the NIS (SAS Institute Inc, Cary, NC).}

\section{RESULTS \\ Patient and Hospital Characteristics}

Patient and hospital demographics are shown in Table 1. Patients undergoing repair were older than those undergoing replacement (median age, 69 years vs 61 years; $P<.0001)$. Of the entire cohort, $61.3 \%$ were female and $71.9 \%$ were white, with no significant difference between the repair and replacement subsets. A substantial number of patients $(42.5 \%)$ were referred for TV surgery nonelectively. Patients in the replacement group more often were referred nonelectively $(P<.0001)$. Moreover, the incidence of endocarditis was significantly higher in the replacement than in the repair group $(14.5 \%$ vs $6.6 \% ; P=.0001)$. Examination of comorbidities between the repair and replacement subsets revealed no significant differences between the 2 groups with respect to congestive heart failure, peripheral vascular disease, cerebrovascular disease, and renal disease. Patients undergoing repair more often had diabetes $(P=.0229)$, pulmonary disease $(P=.0001)$, and a history of myocardial infarction $(P=.0011)$, whereas patients undergoing replacement more often had liver disease $(P=.0001)$.

\section{Trends in Case Mix}

TV repair versus replacement. The total number of admissions for TV operations from 1999 to 2008 was 28,726 . Of these, $72.9 \%(20,951 / 28,726)$ were repairs and $27.1 \%(7775 / 28,726)$ were replacements (Figure 1, A). The total number of TV procedures more than doubled over the 10-year period (1712 cases in 1999 vs 4072 cases in 2008). When stratified by procedure type, although the absolute number of both repair and replacement operations increased over time (Figure 1,A), the TV repair rate increased whereas there was a corresponding decrease in TV replacement rate $(P=.0001)$ (Figure $1, B)$.

Bioprosthetic versus mechanical TV replacement. Overall, tissue valves were used in $46.1 \%$ (3586/7775) of TV replacements with mechanical valves accounted for the remaining 53.9\% (4189/7775). Using the CochranArmitage tests for trends across the years, there was a trend toward increased use of tissue over mechanical valves for TV replacement $(P=.0001)$. Although the absolute number of bioprosthetic and mechanical TV replacements increased over time (Figure 2, A), bioprosthesis use increased from $34.9 \%$ in 1999 to $53.8 \%$ in $2008(P<.0001)$, whereas the use of mechanical prostheses diminished from $65.1 \%$ in 1999 to $46.2 \%$ in $2008(P<.0001)$ (Figure 2, $B)$.

Isolated versus concomitant TV surgery. Isolated TV surgery accounted for $20.0 \%(5736 / 28,726)$ of the total tricuspid cases, whereas TV surgery as a concomitant proceremaining $80.0 \%$. Within the subset of TV repair, $11.8 \%$ of the cases were isolated and $88.2 \%$ were concomitant to other cardiac procedures. Using the Cochran-Armitage tests for trends across the years, there was a trend toward a decreasing rate of isolated TV repairs over time, with a corresponding increasing rate of TV repair performed concomitantly $(P=.0001)$ (Figure $3, A)$. Further trend analysis of TV repair performed as a concomitant procedure revealed an increased rate of TV repair in the setting of mitral repair dure to other cardiac operations accounted for the 
TABLE 1. Characteristics of patients undergoing TV surgery from 1999 to 2008 according to NIS database

\begin{tabular}{|c|c|c|c|c|}
\hline & Overall & Repair & Replacement & $P$ value \\
\hline No. of patients & 28,726 & $20,951(73 \%)$ & $7775(27 \%)$ & \\
\hline Age: mean, median (IQR) & $64.4,67(54-76)$ & $66.0,69(57-76)$ & $59.9,61(48-73)$ & $<.0001$ \\
\hline Female & $17,621(61 \%)$ & $12,918(62 \%)$ & $4703(60 \%)$ & .3931 \\
\hline White & $14,856(72 \%)$ & $10,891(73 \%)$ & $3965(69 \%)$ & .1014 \\
\hline Urban & $17,134(85 \%)$ & $12,786(85 \%)$ & $4348(86 \%)$ & .3203 \\
\hline Admission status & & & & $<.0001$ \\
\hline Elective & $14,621(58 \%)$ & $11,104(60 \%)$ & $3517(52 \%)$ & \\
\hline Urgent/emergency & $10,823(42 \%)$ & $7523(40 \%)$ & $3300(48 \%)$ & \\
\hline \multicolumn{5}{|l|}{ Comorbidities } \\
\hline Previous MI & $1541(5 \%)$ & $1254(6 \%)$ & $287(4 \%)$ & .0011 \\
\hline $\mathrm{CHF}$ & $12,964(45 \%)$ & $9304(44 \%)$ & $3660(47 \%)$ & .1209 \\
\hline PVD & $911(3 \%)$ & $732(4 \%)$ & $180(2 \%)$ & .0645 \\
\hline CVA & $1256(4 \%)$ & $941(4 \%)$ & $315(4 \%)$ & .4666 \\
\hline Pulmonary disease & $5030(18 \%)$ & $3925(19 \%)$ & $1105(14 \%)$ & .0001 \\
\hline Liver disease & $524(2 \%)$ & $240(1 \%)$ & $284(4 \%)$ & .0001 \\
\hline Diabetes & $4437(15 \%)$ & $3373(16 \%)$ & $1064(14 \%)$ & .0229 \\
\hline Renal disease & $2346(8 \%)$ & $1701(8 \%)$ & $645(8 \%)$ & .8262 \\
\hline Endocarditis & $2508(8.7 \%)$ & $1379(6.6 \%)$ & $1129(14.5 \%)$ & .0001 \\
\hline \multicolumn{5}{|l|}{ Hospital characteristics } \\
\hline Teaching hospitals & $20,827(72 \%)$ & $14,813(71 \%)$ & $6014(77 \%)$ & .0023 \\
\hline \multicolumn{5}{|l|}{ Region } \\
\hline Northeast & $6458(22 \%)$ & $4542(22 \%)$ & $1916(25 \%)$ & .0044 \\
\hline South & $7305(25 \%)$ & $5704(27 \%)$ & $1661(21 \%)$ & \\
\hline Midwest & $9300(32 \%)$ & $6898(33 \%)$ & $2402(31 \%)$ & \\
\hline West & $5664(20 \%)$ & $3807(18 \%)$ & $1856(24 \%)$ & \\
\hline \multicolumn{5}{|l|}{ Bed size } \\
\hline Small & $1125(4 \%)$ & $861(4 \%)$ & $263(3 \%)$ & .4304 \\
\hline Medium & $4907(17 \%)$ & $3667(18 \%)$ & $1240(18 \%)$ & \\
\hline Large & $22,695(79 \%)$ & $16,423(78 \%)$ & $6272(81 \%)$ & \\
\hline
\end{tabular}

$T V$, Tricuspid valve; $N I S$, Nationwide Inpatient Sample; $I Q R$, interquartile range; $M I$, myocardial infarction; $C H F$, congestive heart failure; $P V D$, peripheral vascular disease; CVA, cerebrovascular accident.

with or without coronary artery bypass grafting (CABG) $(P=.0001)$, as well as TV repair in the setting of isolated aortic valve replacement $(P=.0464)$ (Figure 3, $B)$, whereas there was a decreasing trend of TV repair performed in the setting of mitral replacement with or without CABG $(P=.0001)$, as well as TV repair in the setting of double valve (aortic and mitral) replacement $(P=.0007)$ (Figure 3,C). Within the subset of TV replacement, 3271 $(42.1 \%)$ of 7775 cases were isolated, whereas 4504 $(57.9 \%)$ of 7775 were concomitant. There were significantly more isolated cases in the replacement subset than in the repair subset $(42.1 \%$ vs $11.8 \% ; P=.0001)$. There was a trend toward increasing rate of isolated TV replacement over time, with a corresponding decreasing rate of TV replacement performed as a concomitant procedure $(P=.0001)$ (Figure 3, $D)$. The majority $(2591 / 4504,57.5 \%)$ of TV replacements performed concomitantly were in the setting of mitral valve replacement with or without CABG.

\section{Hospital Mortality}

Overall hospital mortality for patients undergoing TV surgery was $10.6 \%$. Over time, mortality decreased significantly for both repair and replacement $(P=.0001)$ (Figure 4). Hospital mortality for TV replacement was significantly higher than for TV repair $(13.6 \%$ vs $9.5 \%$; $P=.0001)$. There was no difference in hospital mortality for patients undergoing bioprosthetic versus mechanical TV replacement $(14.2 \%$ vs $13.0 \% ; P=.5023)$. Overall hospital mortality for isolated TV surgery was $9.0 \%$ compared with $11.0 \%$ for concomitant TV surgery $(P=.0520)$. Over time, mortality for isolated TV surgery did not change significantly $(P=.3884)$ whereas mortality for concomitant TV surgery decreased slightly $(P<.0001)$. For the TV repair subset, isolated repair mortality was $7.6 \%$ compared with $9.8 \%$ for repair performed concomitantly $(P=.1438)$. Isolated TV replacement was associated with significantly lower hospital mortality than was TV replacement performed concomitantly with other cardiac procedures $(10.1 \%$ vs $16.1 \% ; P=.0001)$.

Influence of liver disease on mortality. Because the proportion of subjects with liver disease was lower in the repair than in the replacement group ( $1 \%$ vs $4 \% ; P=.0001)$, we specifically compared mortality of patients with or without liver disease stratified by procedure type. The presence of 

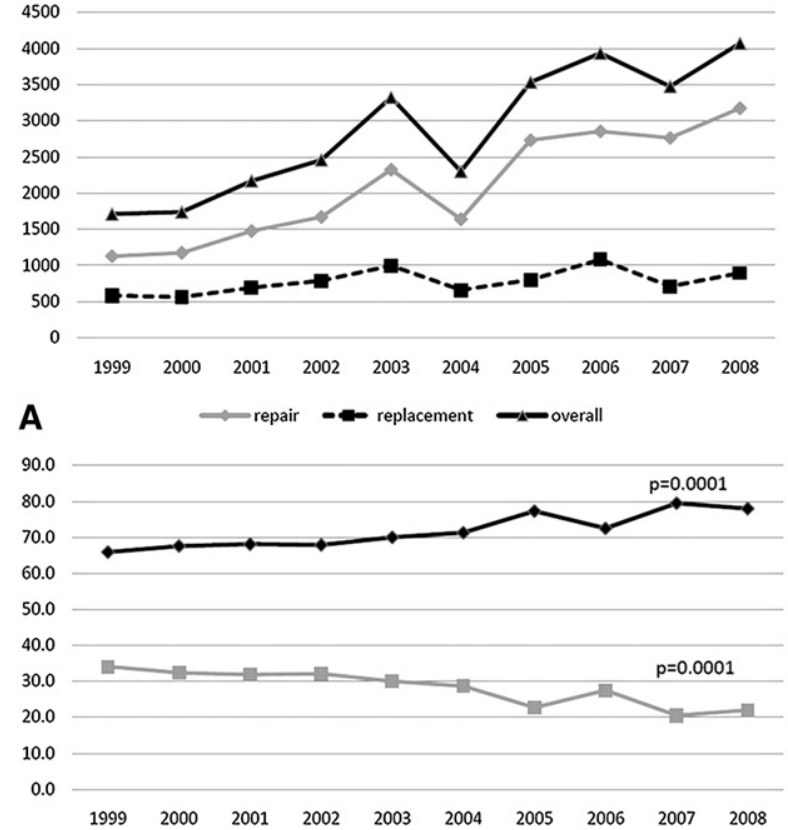

B

FIGURE 1. Trends in tricuspid valve operations from 1999 to 2008 according to the NIS database: A, Total number of tricuspid admissions over time. B, Rates of tricuspid repair and replacement over time.

liver disease was associated with a significantly higher mortality both for patients undergoing repair $(22.85 \%$ [55/240] vs $9.37 \%[1938 / 20,684] ; P=.0011)$ and for those undergoing replacement $(27.04 \%$ [77/284] vs $13.07 \%$ [977/7476]; $P=.0033)$. Consequently, the difference in mortality between the repair and replacement groups was no longer statistically significant among the subset of patients with liver disease undergoing TV surgery $(22.85 \%$ [55/240] vs $27.04 \%$ [77/284]; $P=.6225$ ). When we compared mortality for repair versus replacement among patients without liver disease, mortality for replacement remained significantly higher $(13.07 \%$ [977/7476] vs $9.37 \%$ [1938/ 20,684]; $P=.0001)$.

Length of stay. Median length of stay for TV repair was significantly less than for replacement (12 days vs 15 days; $P=.0001)$. Furthermore, within the repair subset, there was no difference in median length of stay for isolated versus concomitant procedures (11 days vs 12 days; $P=.4283$ ). Within the replacement subset, median length of stay for bioprosthetic and mechanical valves was similar (14 days vs 15 days; $P=.4497$ ) as it was for isolated versus concomitant TV replacement (15 days for both).

Length of stay was initially examined as a continuous outcome but, because of distributional characteristics, was subsequently dichotomized using a median split (length of stay, 12 days). Therefore, prolonged hospitalization was defined as length of stay equal to or greater than 12 days.
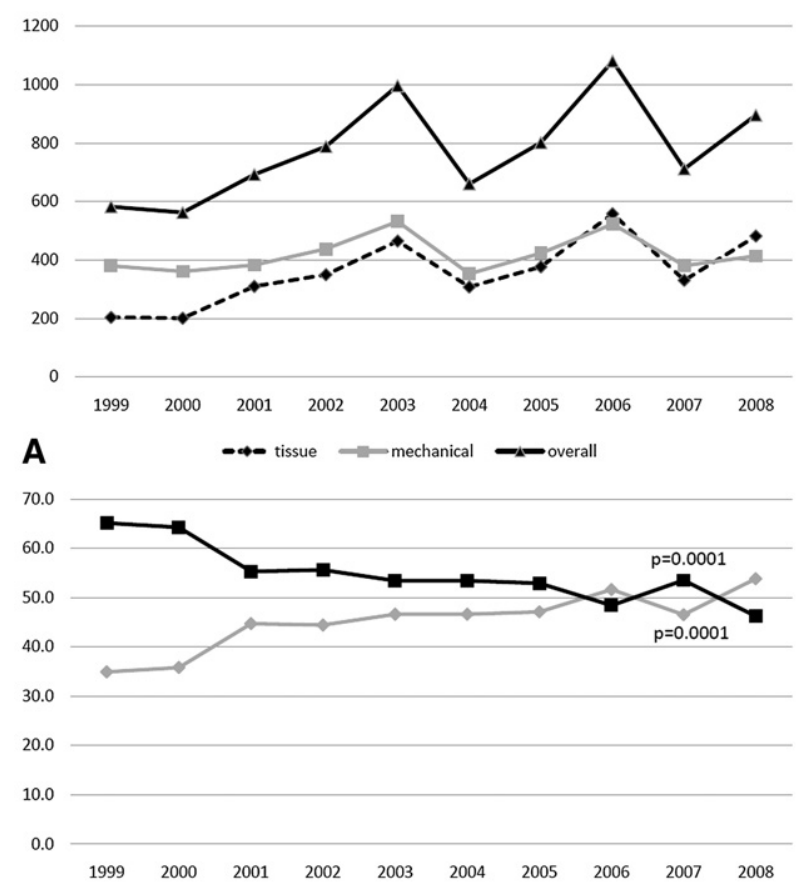

B

FIGURE 2. Trends in tricuspid valve replacement from 1999 to 2008 according to NIS database: A, Total number of tricuspid replacements over time. B, Rates of bioprosthesis versus mechanical tricuspid valve replacement over time.

Overall, there was an increasing trend of prolonged hospitalization over time for both the repair $(P<.0001)$ and replacement $(P<.0001)$ subsets.

Pacemaker implantation. Overall, pacemaker implantation occurred in $11.6 \%$ of the patients undergoing TV valve surgery. As expected, the need for a postoperative pacemaker was significantly higher in the replacement group than in the repair group $(17.2 \%$ vs $9.5 \% ; P=.0001)$.

\section{DISCUSSION}

Despite recent advances in cardiac surgery, TV intervention remains associated with high morbidity and mortality. ${ }^{6}$ Considering that TV surgery is relatively rare, many previous series come from high-volume institutions and may not be an accurate depiction of national trends. Therefore, we undertook this investigation to analyze the trends in demographics, case mix, procedure selection, and hospital outcomes of patients undergoing TV surgery over the period from 1999 to 2008 using data from the NIS. The NIS database is the largest all-payer database in the United States and has been used extensively for analysis of trends in health care utilization, access, charges, quality, and outcomes for both research and policy making (HCUP databases).

Consistent with the decreasing mortality for aortic and mitral valve surgery, we found a similar trend in TV surgery. 


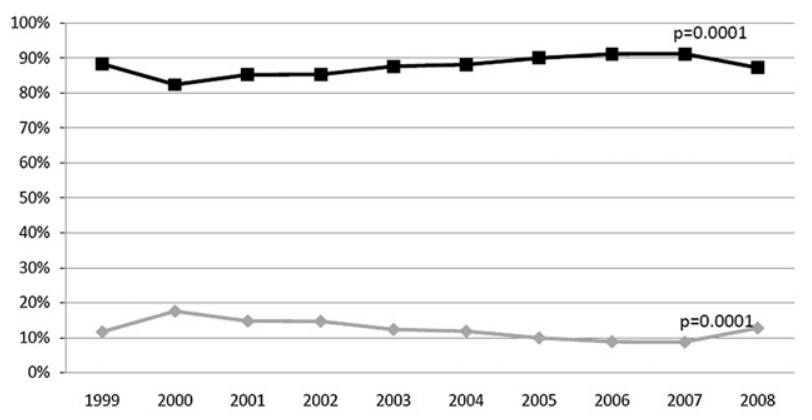

A

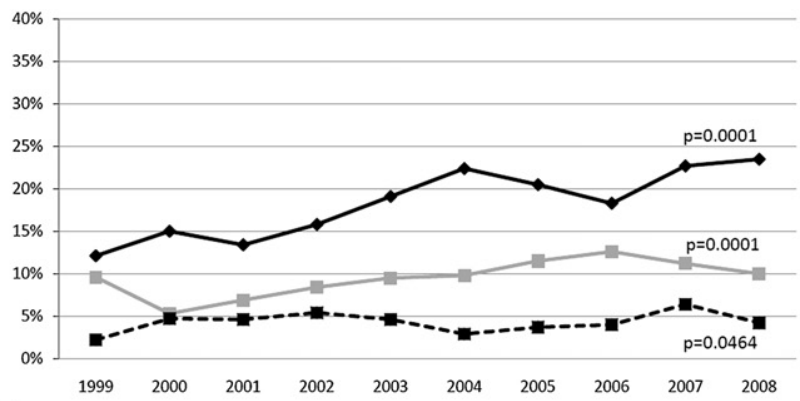

B $\rightarrow$ w/Mitral Repair $\rightarrow$ w/ CABG+Mitral Repair $\quad-m-$ w/ Aortic Replace

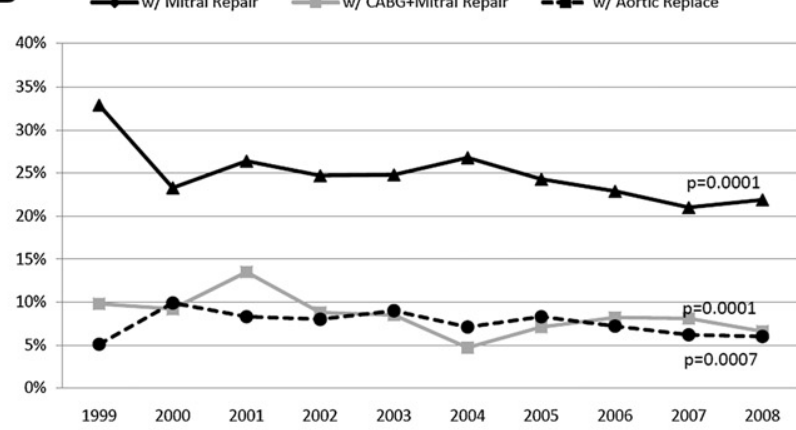

C $-w /$ Mitral Replace $-w$ w/ CABG+Mitral Replace - - w/ Aortic Replace+Mitral Replace

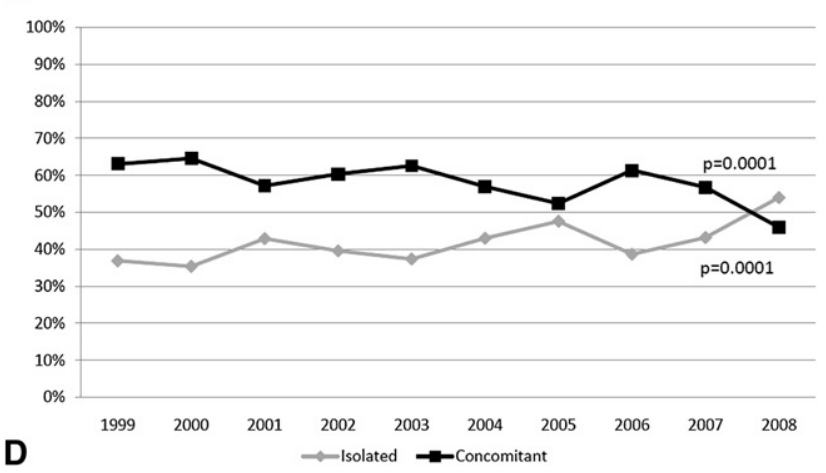

FIGURE 3. Trends in isolated versus concomitant tricuspid surgery from 1999 to 2008 according to the NIS database: A, Trends in isolated versus concomitant tricuspid valve repair. B, Increasing trends of tricuspid repair performed concomitantly. $\mathrm{C}$, Decreasing trends in concomitant procedures with tricuspid repair. D, Trends in isolated versus concomitant tricuspid valve replacement over time. $C A B G$, Coronary artery bypass grafting.

Although overall hospital mortality decreased over time, across the 10-year period, it was significantly higher for replacement than for repair. It appears that this difference in

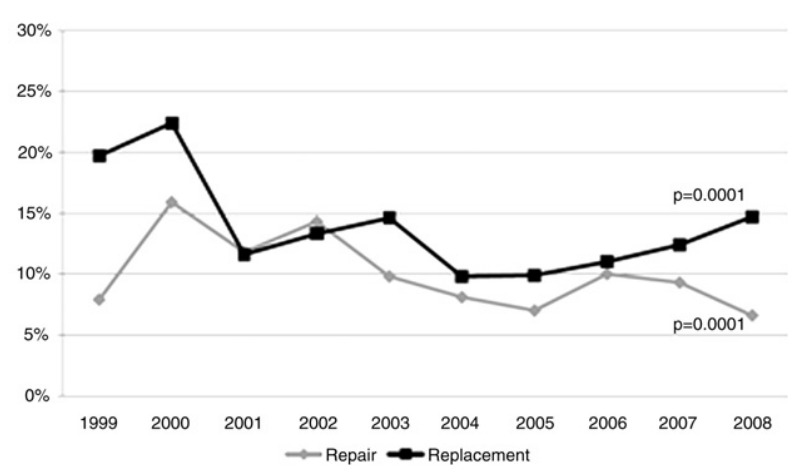

FIGURE 4. Trends in tricuspid mortality over time.

mortality is primarily driven by the higher mortality of concomitant TV replacement in the setting of other cardiac procedures (more than half of these being concomitant mitral valve replacement with or without $\mathrm{CABG}$ ). Lower mortality for repair versus replacement has also been reported in mitral valve surgery. ${ }^{7-9}$ Although within the subset of TV repair there was no difference with respect to mortality of isolated versus concomitant procedure, in the TV replacement subset, concomitant procedures had significantly higher mortality than did isolated TV replacement $(16.1 \%$ vs $10.1 \%$ ). Consequently, this identifies an important area for future improvement in the care of patients with valvular heart disease.

Compared with aortic and mitral valve surgery, TV surgery remains associated with significantly higher mortality. Filsoufi and associates ${ }^{10}$ reported a $22 \%$ mortality for a small heterogeneous series of 81 patients undergoing TV replacement. In their series, $72 \%$ of the cases were reoperations. ${ }^{10}$ Ratnatunga and colleagues ${ }^{11}$ showed a $17.3 \%$ 30-day mortality for a series of patients from the United Kingdom valve registry, and Kaplan and associates ${ }^{5}$ reported an overall hospital mortality of $24.5 \%$. Our overall hospital mortality is lower than what has been reported by others. Several explanations exist for this discrepancy. Because our mortality for concomitant TV replacement was significantly higher than that for isolated replacement, discrepancy in results may be due to differences in relative proportions of patients with isolated versus concomitant surgery in different series. Furthermore, results from various authors may vary depending on the cause of TV disease. For example, Singh and coworkers ${ }^{3}$ studied a group of 250 patients who underwent TV surgery for organic valve disease from 1979 to 2003. This group (not separated by isolated or concomitant) had a hospital mortality rate of $4 \%$ for TV repair and $22 \%$ for TV replacement. In contrast, mortality for TV replacement for carcinoid heart disease has been reported as high as $63 \% .^{12}$ The overall hospital mortality rate for TV surgery performed on 328 patients with rheumatic disease was $6.4 \%$ for repair and $19.3 \%$ for replacement, ${ }^{13}$ whereas TV surgery concomitantly 
with either aortic or mitral valve operation carried a hospital mortality rate of $8.1 \% .^{14}$

The presence of liver disease confers an excessive risk of mortality in TV surgery $(>20 \%)$, regardless of procedure selection (repair vs replacement). Although in the group of patients undergoing replacement the incidence of liver disease was higher, it appears that the difference in mortality between the repair and replacement groups is only partly explained by the higher incidence of liver disease in the replacement group. The difference in mortality rates between repair and replacement for those without liver dysfunction was $3.7 \%$ whereas the difference for those with liver dysfunction was $4.19 \%$. Others have used The Model for End-Stage Liver Disease score to stratify patients with liver disease undergoing TV surgery and found that this score predicts mortality in this subset of patients and may be used as a simple method for risk stratification. ${ }^{15}$

Published TV repair series typically have included either patients with isolated TV disease or patients undergoing other cardiac procedures, such as mitral valve surgery, during which TV repair was performed as a concomitant procedure. Our investigation provides the largest series comparing trends and outcomes for these 2 subsets of patients. We found that there was an overall increase in concomitant TV repair over time in our case mix. We further analyzed this overall trend and found an increased number of TV repairs performed concomitantly with mitral repair with or without CABG. This is consistent with recent literature, suggesting that there is a specific subset of patients with TV regurgitation of even moderate degree in the setting of mitral valve disease that may benefit from TV intervention. ${ }^{16,17}$ Furthermore, the TV disease in these cases is often amenable to repair. ${ }^{16,17}$ Because the NIS database contains discharge data only from the initial hospitalization, longitudinal follow-up studies are needed to examine how an increase in TV repairs affects long-term repair durability and survival. Although within the NIS database we cannot establish the cause of TV disease (functional vs organic), it appears that the increasing trend of concomitant TV repair, especially in the setting of mitral valve surgery, reflects a more aggressive approach to TV disease. Consequently, TV repairs performed concomitantly with mitral replacement with or without CABG were shown to decrease over time. This finding is consistent with the general trend of increasing valve repair over replacement as the procedure of choice for surgical correction of mitral valve disease. ${ }^{7}$ The advantages of mitral repair over replacement have been widely established. ${ }^{7}$ Similar to this trend in mitral surgery, we found that there was a significant increase in TV repair rates over time, with a corresponding decrease in the rates of replacement. TV regurgitation is a widely known sequela of longstanding mitral valve disease. Consequently, inasmuch as recent guidelines on the management of valvular heart disease recommend earlier intervention on the mitral valve, there may be a corresponding decrease in secondary TV disease in the future. ${ }^{18}$

In contrast to the trend in TV repair, we found a decreasing trend of concomitant TV replacement. Interestingly, the majority of these were performed in the setting of mitral valve replacement with or without CABG. It is possible that mitral disease not amenable to repair is associated with a corresponding TV disease also not amenable to repair. The NIS database does not provide detailed information of the etiology (eg, degenerative vs rheumatic) or type (stenosis vs regurgitation) of organic TV disease, limiting our ability to draw any definite conclusions with respect to this finding. Certainly, in the overall population, the presence of endocarditis appears to increase the risk of undergoing replacement.

Multiple investigations have addressed the ongoing debate with respect to the ideal type of prosthesis in the tricuspid position, showing similar long-term survival, reoperation rates, and valve-related complications. ${ }^{5,10,19,20}$ In agreement with previously published literature, we found similar length of stay and mortality for bioprosthetic and mechanical valves. Nevertheless, there was a significant increase in the use of bioprosthetic valves from $34.9 \%$ in 1999 to $53.8 \%$ in 2008 . This finding is likely due to the body of literature establishing equivalence between bioprosthetic and mechanical valves, therefore offering the opportunity to avoid anticoagulation-related complications. This is consistent with the finding that there was an increasing trend of isolated TV replacement, as choice of prosthesis during concomitant TV replacement may be determined by the need for anticoagulation with respect to the primary surgical indication.

Our study has several limitations. The NIS is a stratified probability sample of state inpatient databases including data on roughly $20 \%$ of hospital admissions in the United States. Therefore, our data were weighted to make them more applicable to the entire inpatient population. The purpose of administrative databases is to gather data for billing purposes and can be limited by erroneous coding. However, the HCUP quality control measures should minimize these possibilities (HCUP databases). Furthermore, the hard clinical end points used in our analysis are difficult to miscode.

There are several unaccounted for factors that may have influenced surgeon decision to perform TV repair versus replacement. These include information regarding right ventricular function, pulmonary hypertension, functional versus organic TV disease, regurgitation versus stenosis as the indication for surgery, and severity of regurgitation, especially for TV repair or replacement performed concomitantly with other cardiac procedures. Nevertheless, we provide the largest series of TV surgery, including comparisons on repair versus replacement, isolated versus concomitant surgery, and bioprosthetic versus mechanical TV replacement. The NIS database only contains data on the initial hospitalization 
and therefore clinical or echocardiographic follow-up information is not available. Consequently, our study cannot comment on long-term survival after TV surgery, reoperation rates, and/or TV repair durability.

Several strengths are noted with respect to data from the NIS. Participation is not voluntary and sampling is weighted to reflect national averages. Furthermore, the NIS is the largest all-payer database in the United States and some of its limitations are offset by large patient volumes, hard clinical end points, and the opportunity to explore real world community data, making our findings widely applicable across hospitals in the United States.

We thank Joshua DiGennaro for assistance with manuscript table and figure preparation and Jean Long for assistance with manuscript submission and editing.

\section{References}

1. Gammie JS, Sheng S, Giffith BP, Peterson ED, Rankin JS, O'Brien SM, et al. Trends in mitral valve surgery in the United States: results from The Society of Thoracic Surgeons adult cardiac surgery database. Ann Thorac Surg. 2009; 87:1431-7.

2. Brown JM, O'Brien SM, Wu C, Sikora JA, Griffith BP, Gammie JS. Isolated aortic valve replacement in North America comprising 108,687 patients in 10 years: changes in risks, valve types, and outcomes in The Society of Thoracic Surgeons national database. J Thorac Cardiovasc Surg. 2009;137:82-90.

3. Singh SK, Tang GH, Maganti MD, Armstrong S, Williams WG, David TE, et al. Midterm outcomes of tricuspid valve repair versus replacement for organic tricuspid disease. Ann Thorac Surg. 2006;82:1735-41.

4. Moraca RJ, Moon MR, Lawton JS, Guthrie TJ, Aubuchon KA, Moazami N, et al. Outcomes of tricuspid valve repair and replacement: a propensity analysis. Ann Thorac Surg. 2009;87:83-8.

5. Kaplan M, Kurt MS, Demirtas MM, Cimen S, Ozler A. Prosthetic replacement of tricuspid valve: bioprosthetic or mechanical. Ann Thorac Surg. 2002;73:467-73.

6. Kratz JM, Crawford FA, Stroud MR, Appleby DC, Hanger KH. Trends and results in tricuspid valve surgery. Chest. 1985;88:837-40.

7. Barnett SD, Ad N. Surgery for aortic and mitral valve disease in the United States: a trend of change in surgical practice between 1998 and 2005. J Thorac Cardiovasc Surg. 2009;197:1422-9.
8. Zhou YX, Leobon B, Berthoumieu P, Roux D, Glock Y, Mei YQ, et al. Long-term outcomes following repair or replacement in degenerative mitral valve disease. Thorac Cardiovasc Surg. 2010;58:415-21.

9. LaPar DJ, Hennessy S, Fonner E, Kern JA, Kron IL, Ailawadi G. Does urgent or emergent status influence choice in mitral valve operations? An analysis of outcomes from the Virginia Cardiac Surgery Quality Initiative. Ann Thorac Surg. 2010;90:153-60

10. Filsoufi F, Anyanwu AC, Salzberg SP, Frankel T, Cohn LH, Adams DH. Longterm outcomes of tricuspid vlave replacement in the current era. Ann Thorac Surg. 2005;80:845-50.

11. Ratnatunga CP, Edwards MB, Dore CJ, Taylor KM. Tricuspid valve replacement: UK Heart Valve Registry mid-term results comparing mechanical and biological prostheses. Ann Thorac Surg. 1998;66:1940-7.

12. Robiolio PA, Rigolin VH, Harrison JK, Lowe JE, Moore JO, Bashore TM, et al. Predictors of outcome of tricuspid valve replacement in carcinoid heart disease. Am J Cardiol. 1995;75:485-8.

13. Bernal JM, Ponton A, Diaz B, Llorca J, Garcia I, Sarralde A, et al. Surgery for rheumatic tricuspid valve disease: a 30-year experience. J Thorac Cardiovasc Surg. 2008; 136:476-81.

14. Bernal JM, Gutierrez-Morlote J, Llorca J, San Jose JM, Morales D, Revuelta JM. Tricuspid valve repair: an old disease, a modern experience. Ann Thorac Surg. 2004;78:2069-75.

15. Ailawadi G, Lapar DJ, Swenson BR, Siefert SA, Lau C, Kern JA, et al. Model for end-stage liver disease predicts mortality for tricuspid valve surgery. Ann Thorac Surg. 2009;87:1460-7.

16. Van de Veire NR, Braun J, Delgado V, Versteegh MI, Dion RA, Klautz RJ, et al Tricuspid annuloplasty prevents right ventricular dilatation and progression of tricuspid regurgitation in patients with tricuspid annular dilatation undergoing mitral valve repair. J Thorac Cardiovasc Surg. 2011;141:1431-9. Epub 2010 Sep 15.

17. Calafiore AM, Iacò AL, Romeo A, Scandura S, Meduri R, Varone E, et al. Echocardiographic-based treatment of functional tricuspid regurgitation. J Thorac Cardiovasc Surg. 2011;142:308-13. Epub 2010 Dec 15.

18. Bonow RO, Carabello BA, Chatterjee K, de Leon AC Jr, Faxon DP, Freed MD, et al. 2008 focused update incorporated into the ACC/AHA 2006 guidelines for the management of patients with valvular heart disease: a report of the American College of Cardiology/American Heart Association Task Force on Practice Guidelines. J Am Coll Cardiol. 2008;52:1-142.

19. Rizzoli G, De Perini L, Bottio T, Minutolo G, Thiene G, Cassarotto D. Prosthetic replacement of the tricuspid valve: biological or mechanical? Ann Thorac Surg. 1998;66:62-7.

20. Rizzoli G, Vendramin I, Nesseris G, Bottio T, Guglielmi C, Schiavon L. Biological or mechanical prosthesis in tricuspid position? A meta-analysis of intrainstitutional results. Ann Thorac Surg. 2004;77:1607-14. 